\title{
Direct Current Helium Plasma for In vivo Delivery of Plasmid DNA Encoding Eythropoietin to Murine Skin
}

\author{
Mark J. Jaroszeski, a,b, ${ }^{\text {, }}$ Taryn Harvey-Chapman, ${ }^{\mathrm{a}, \mathrm{b}}$ Andrew Hoff,, ${ }^{\mathrm{b}, \mathrm{c}}$ Reginald \\ Atkins, ${ }^{\mathrm{a}, \mathrm{b}}$ \& Richard J. Connollya,b \\ ${ }^{\mathrm{a} D e p t}$ of Chemical and Biomedical Engineering, University of South Florida, College of Engineering,

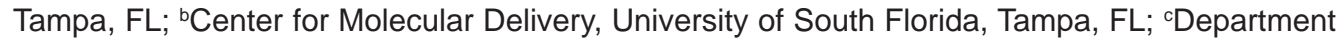 \\ of Electrical Engineering, University of South Florida College of Engineering, Tampa, FL \\ *Address all correspondence to: Mark J. Jaroszeski, Ph.D., Associate Professor, University of South Florida, Department \\ of Chemical and Biomedical Engineering, ENB 118, 4202 E. Fowler Avenue, Tampa, FL 33620; Tel.: (813) 974-4662; \\ Fax: (813) 974-4662, E-mail: mjarosze@usf.edu
}

\begin{abstract}
The use of electric fields in vivo to deliver DNA, called electroporation, has the potential to broadly impact vaccination and disease treatment. The evidence for this has emerged from a large number of recently completed and ongoing clinical trials. The methods for applying electric fields to tissues traditionally involve contact between metal electrodes and the tissue. In this study, we investigated the use of helium plasma as a noncontact method for electrically treating tissue in a manner that results in the uptake and expression of foreign DNA in murine skin. More specifically, our goal was to demonstrate that DNA encoding a model-secreted protein could be delivered, detected in the blood, and remain functional to produce its known biological effect. Murine erythropoietin (EPO) was the model-secreted protein. Results clearly demonstrated that an intradermal DNA injection followed by plasma treatment for 2 min resulted in elevated levels of EPO in the blood and corresponding hemoglobin increases that were statistically significant relative to DNA injection alone.
\end{abstract}

KEY WORDS: gene delivery, gene therapy, DNA delivery, electroporation

\section{INTRODUCTION}

Electrogene therapy offers the promise of safe and effective disease treatment and has steadily progressed from the research stage to the clinic during the past 20 years. ${ }^{1-3}$ It is clear that in the future, this type of gene delivery technology will broadly impact vaccination, treatment of metabolic diseases, and cancer therapy. Virtually all research and clinical studies have relied on the application of direct current (DC) electric pulses to target tissues that have been injected with plasmid DNA. Increased cellular DNA uptake is directly related to the physical forces that result after exposure to electrical fields. The underlying mechanisms enabling electrogene therapy are not yet fully understood. Uptake may be related to transient defects/electropores that form in cell membranes ${ }^{4-6}$ or by processes initiated by electric field exposure. ${ }^{7,8} \mathrm{~A}$ common characteristic of nearly all electrogene therapy procedures is the application of DC electric pulses administered to tissues with electrodes that contact the target tissue or needles that penetrate the tissue. While this contact-dependent approach has been highly successful in enhancing mo- 
lecular delivery, it does have some drawbacks, such as involuntary muscle stimulation and transient discomfort, and can result in tissue damage at the treatment site. ${ }^{9-11}$

An alternative means of applying electric fields to tissue is by depositing charge in the form of ionized/charged gas molecules, or plasma, onto the surface of a treatment site. Plasma applicators are not in direct contact with the target tissue during deposition. In fact, plasma is typically generated millimeters to centimeters away from the tissue. Charge emanates from the generator that typically has a pen-sized applicator similar to most sets of electroporation electrodes. The use of ionized discharges for molecular delivery is relatively recent. Ionized discharges were first proven to be capable of enhancing the delivery of small molecules and plasmid DNA in vitro. ${ }^{12-16}$ Subsequently, they have been used to increase the uptake of plasmid DNA encoding for reporter molecules and antigens in murine skin. ${ }^{17-21}$ In addition, plasma discharges have been used to deliver plasmid DNA encoding cytokines to subcutaneous melanomas in mice. ${ }^{22}$ Plasma sources for in vitro delivery have been driven by both alternating current and DC. Those used for in vivo delivery have relied on DC signals. Plasma generators used in both settings have been nonthermal.

The potential clinical applications of plasma-based DNA delivery are abundant. Previous studies have shown that local levels of luciferase increase after plasma has been applied to skin covering intradermally injected plasmid DNA encoding the reporter gene. Luciferase levels in treated skin have been as much as 30 times higher than those resulting after injecting DNA alone. ${ }^{17,20,21}$ Similarly, cellular and humoral immune responses have been elicited in response to delivering plasmid DNA encoding the HIV antigen gp120 locally to a small patch of murine skin. ${ }^{17,18}$ These studies demonstrated the capability of plasma to enhance subcutaneous plasmid DNA delivery. However, the capacity for the technique to result in increased expression of a systemically circulating protein that has effects distant to the delivery site has not been examined. Therefore, the purpose of this study was to determine suitable plasma conditions to enhance delivery of plasmid DNA in murine skin and use those conditions to deliver plasmid DNA encoding erythropoietin (EPO). Uptake of this biologically active plasmid could be determined analyzing systemic EPO levels in the serum and assessing hemoglobin levels.

\section{MATERIALS AND METHODS}

\section{A. Animal Model and Anesthesia}

Six-week-old female BALB/c mice (National Institutes of Health; Bethesda, MD) were used as the model system for this study. All procedures relating to this study were performed in accordance with a protocol approved by the University of South Florida Institutional Animal Care and Use Committee. The left flank skin of the animals was selected as the site for delivery. Before treatment, hair was removed from the flank of each animal using standard clippers. Mice were anesthetized during all DNA delivery procedures and imaging. Anesthesia was induced by placing the animals in an induction chamber filled with $2 \%$ isoflurane in oxygen supplied by a calibrated vaporizer. After 
induction, animals were fitted with a scavenged rodent mask to maintain anesthesia during the delivery procedure. Animals were placed on a $32^{\circ} \mathrm{C}$ warming pad during the DNA delivery procedure to help maintain body temperature.

\section{B. Plasma Source}

The plasma source for this study has been described in detail elsewhere. ${ }^{13,20}$ Principal components included a hollow, 15-cm-long nylon dowel that served as the plasma applicator. The applicator had a 1-cm diameter with a wall thickness of $2 \mathrm{~mm}$ (Fig. 1A). The upper end of the nylon plasma applicator had a fitting so that helium gas could be plumbed to it, enabling the gas to flow through the applicator. A helium flow rate of $15 \mathrm{~L} / \mathrm{min}$ was used for this study; it was controlled by a standard rotameter (PMR1-010280 with a 014-96-N flow tube; Cole-Parmer Instruments; Vernon Hills, IL). The gas source was high-purity helium (Airgas; Tampa, FL) from a standard cylinder. The upper end of the plasma applicator also had a 5-mW red laser (Instapark, Inc.; Santa Fe Springs, CA). The laser was aligned with the center of the hollow, nylon dowel to provide a means to aim the plasma precisely to the target tissue. The lower end of the plasma applicator housed an annular stainless-steel electrode with a 3.5-mm internal diameter (Fig. 1B) that was connected to a high-voltage DC generator (Spellman CZE2000; Hauppauge, NY).

Voltage, polarity, maximum current, and exposure time were adjusted for treatment using a program written in LabView (National Instruments Corporation; Austin, TX). The software also performed real-time monitoring and logging of both the current and potential at the high-voltage electrode. The plasma applicator was fixed $1 \mathrm{~cm}$ above the treatment site of all animals in this study and was set for a potential of either $-8 \mathrm{kV}$ or

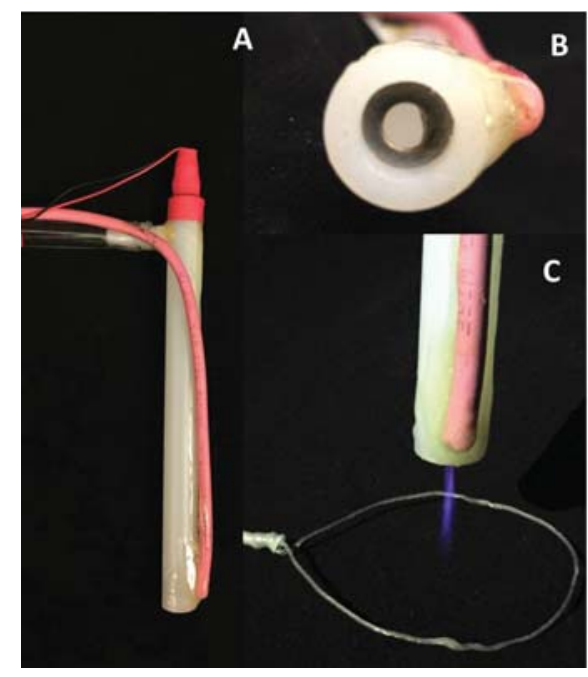

FIG. 1: (A) Side view of the plasma applicator; (B) high-voltage annular electrode; (C) afterglow created by the plasma applicator when operating at $+8 \mathrm{kV}$

Volume 7, Issue 3, 2017 
$+8 \mathrm{kV}$. Under these operating conditions, approximately $15 \mu \mathrm{A}$ of current flowed from the generator to the plasma applicator, which created a visible plume of ionized gas. This plume, shown in Fig. 1C, was applied to the skin by first laying the 22-gauge bare wire ring shown in the figure around the treatment site. One end of an insulated wire was connected to the ring. The other end of this insulated wire, not shown, was connected to ground potential after passing through a $1.5-\mathrm{G} \Omega$ resistor. This arrangement was determined to produce higher expression and result in a plume with increased stability when operated in a room with minor air currents. ${ }^{13,20}$ Plasma was applied for times ranging from $30 \mathrm{~s}$ to $20 \mathrm{~min}$.

\section{Electroporation Apparatus}

Electroporation was performed to generate data for comparison. A multielectrode array (MEA) consisting of 16 electrodes in a $4 \times 4$ array (Fig. 2) was applied to the skin surface above the DNA injection site. The distance between electrode centers was 2.5 $\mathrm{mm}$, and electrodes were $0.5 \mathrm{~mm}$ in diameter. A total of 72 pulses were applied to the skin using the electrode with a defined sequence that treated using nine subsets of four electrodes to pulse the area beneath the array in a piecewise manner. Each rectangular DC pulse applied had field strength of $175 \mathrm{~V} / \mathrm{cm}$ and pulse duration of $150 \mathrm{~ms}$ that were determined to provide optimal plasmid DNA delivery. ${ }^{23,24}$ A custom-built generator and aplicator was used to administer the pulses in the appropriate sequence with a $150-\mathrm{ms}$ delay between successive pulses.

\section{DNA Deliveny to Murine Skin}

Delivery with plasma and electroporation was performed by first intradermally injecting plasmid DNA, in saline, using a 33-gauge needle (SteriJect; TSK Laboratories; Vancouver, BC) into the skin of the shaved left flank. Two different plasmids were used for

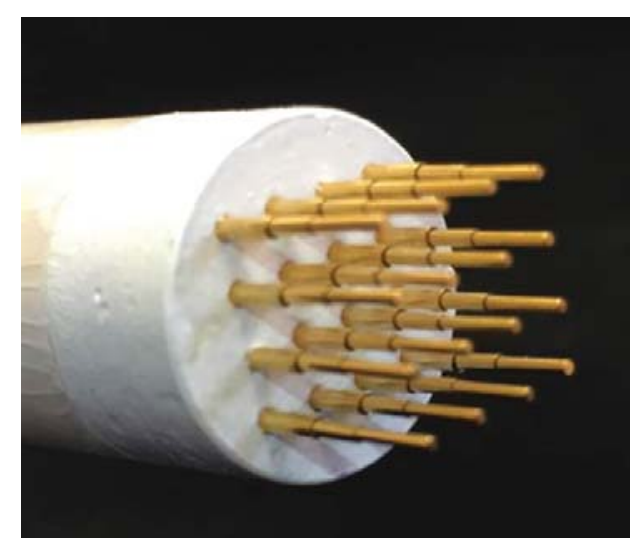

FIG. 2: MEA that was used for electroporative DNA delivery 
plasma-based delivery: One encoded the reporter luciferase (gWiz luciferase; Aldevron; Fargo, ND) and the other encoded murine EPO (gWiz EPO, Aldevron; Fargo, ND). Electroporation was only performed with plasmid DNA encoding EPO as a basis for comparison of the biological response. All plasmids were delivered at a dose of $100 \mu \mathrm{g}$ suspended in $50 \mu \mathrm{L}$ saline. Following injection, the skin overlaying the injection site was immediately treated with either plasma or electroporation, as described above, to complete the delivery procedure.

\section{E. Plasma Dose Response using luciferase Encoding Plasmid}

Experiments to elucidate the luciferase expression profile in skin in response to plasma treatment time and polarity included 12 treatment groups. The first group received no treatment. A second group received only an injection of plasmid DNA encoding luciferase with no plasma treatment. Five groups received plasma generated with $+8 \mathrm{kV}$ applied to the annular electrode immediately following DNA injection. These groups received treatment for $0.5,2,5,10$, or $20 \mathrm{~min}$. The remaining groups received plasma treatment for the same time but $-8 \mathrm{kV}$ was used to create the plasma. Luciferase expression in the flank skin was quantitated on days $0,2,4,7,14,21$, and 30. Each group contained 16 animals; treatment was conducted in a series of four identical experiments with four animals per treatment group.

\section{F. Delivery of EPO Encoding Plasmid and Biological Response}

The in vivo response to plasma-delivered murine EPO encoding DNA was established by treating five groups of animals. The first received no treatment. The second received only an intradermal injection of the plasmid encoding murine EPO. The remaining three treatment groups received plasmid DNA injections followed by optimal electroporation, positive plasma for an experimentally determined time, or negative plasma for an experimentally determined time. All treatment groups were comprised of 12 animals. Serum EPO levels in all animals were quantitated over a 40-day period using an enzyme-linked immunosorbent assay (ELISA). The response to increased EPO levels is increased hematocrit or red blood cell concentration. Post-treatment analysis of hemoglobin levels in the blood was used as a surrogate for actual hematocrit levels because it required very little blood as compared to hematocrit determinations. Thus, periodic measurements could be conducted on each animal without affecting their health during the course of the study.

\section{G. Evaluation of DNA Expression}

\section{Luciferase Expression}

Anesthetized animals were each given an intraperitoneal injection of $150 \mathrm{mg} / \mathrm{kg}$ D-luciferin (Caliper Life Sciences; Hopkinton, MA) prepared in phosphate-buffered saline. 
Fifteen minutes after injection, each animal was placed into a Xenogen IVIS 200 imaging system (Caliper Life Sciences; Hopkinton, MA) to quantitate light produced in the flank skin by the expressed luciferase and injected luciferin. Photon flux from each animal was calculated using Living Image Software (PerkinElmer, Inc.; Shelton, CT) incorporated into the IVIS 200 system. This noninvasive system allowed the luciferase levels in the same groups of animals to be determined periodically during a 30 -d period.

\section{EPO Expression}

EPO, a hormone produced naturally by the kidneys, stimulates red blood cell production in bone marrow. Increased numbers of red blood cells results in increased hematocrit, which is the ratio of red cell volume to the volume of whole blood. Hemoglobin levels are increased proportionally with hematocrit, because red blood cells contain hemoglobin. When the plasmid encoding EPO was expressed, the resulting EPO in the bloodstream acted systemically. Both EPO protein levels and resulting hemoglobin concentrations were monitored and directly related to expression of the exogenous EPO gene. ${ }^{25}$ In these experiments, plasma EPO and hemoglobin levels were monitored using blood samples taken submandibularly on days 0 (before treatment), 10, 20, and 30 . Terminal samples were taken on day 40 by cardiac puncture.

When blood was drawn from the animals, hemoglobin levels were determined by adding $10 \mu \mathrm{L}$ of blood to a microcuvette (HemoCue AB; Ängelholm, Sweden) and immediately reading hemoglobin levels using a HemoCue $\mathrm{Hb} 201^{+}$instrument (HemoCue AB; Ängelholm, Sweden). The remaining blood was collected in serum-separating tubes (BD Microtainer; Becton Dickinson and Company; Franklin Lakes, NJ). Serum was isolated from blood samples by centrifugation at $2000 \mathrm{~g}$ for $10 \mathrm{~min}$ at $4{ }^{\circ} \mathrm{C}$. Immediately following serum isolation, the samples were diluted twofold and assayed with Quantikine ELISA Mouse EPO kits (R\&D Systems; Minneapolis, MN). The optical density of each sample was determined by reading the ELISA plates with a 450-nm filter on a plate reader (FLx800; BioTek Instruments, Inc.; Winooski, VT). A standard curve was generated for each plate by using dilutions of a mouse EPO standard to determine concentrations of the EPO in the samples.

\section{H. Statistical Analysis}

We used a nonparametric Kruskal-Wallis test along with a Dunn's post-test to compare results from different treatment groups. A 95\% confidence level was used for all tests.

\section{RESULTS}

\section{A. Plasma Dose Response using luciferase Encoding Plasmid}

To determine a plasma treatment time that maximized subsequent expression, an experiment was conducted using luciferase-expressing plasmid DNA. This experiment 


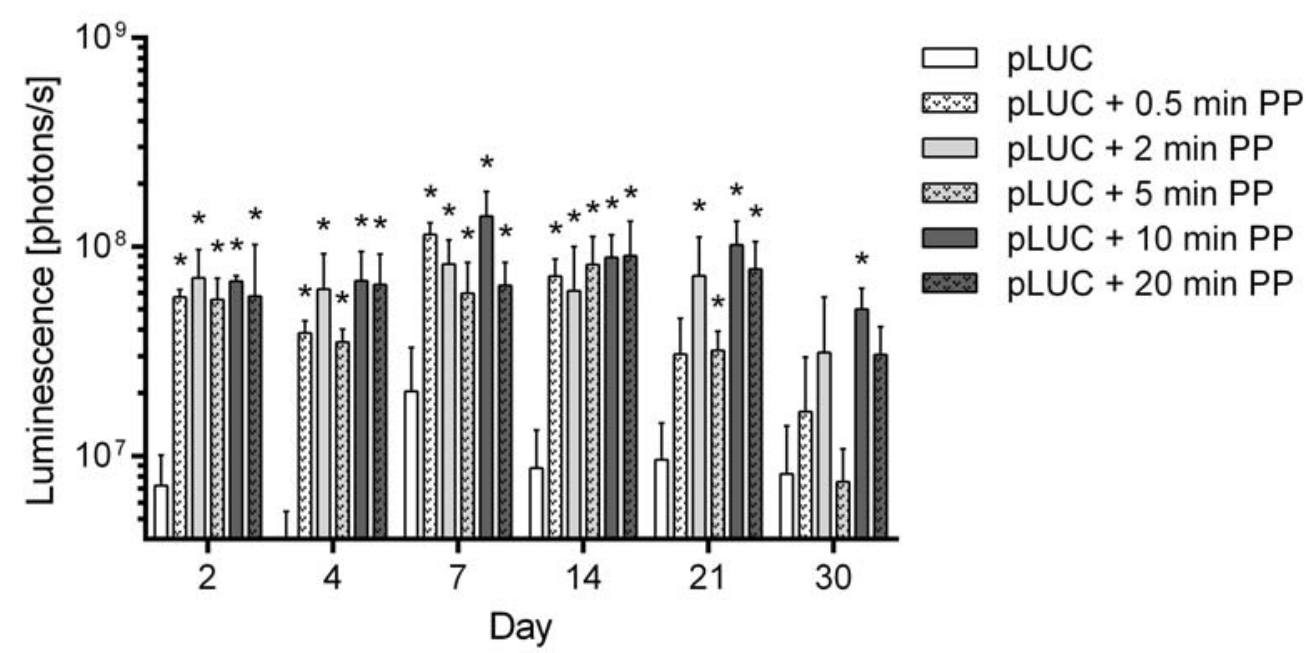

FIG. 3: Long-term luminescence data for animals that had positive polarity plasma used for DNA delivery. pLUC, Plasmid encoding luciferase administered intradermally; PP, positive plasma; $x x$ min, treatment time with plasma; $n=16$ animals per treatment group. Error bars represent the standard error of the mean. *Statistical significance relative to pLUC injection alone on the same follow-up day

used plasma treatment times of $0.5,2,5,10$, and 20 min for plasma generated using a positive potential and a negative potential. Groups of animals that received no treatment and DNA only were included in the experiment. Figure 3 shows resulting luminescence data during a $30-\mathrm{d}$ period for treatment groups that had positive plasma used for DNA delivery. Figure 4 shows the analogous data for negative polarity plasma.

For animals that received positive polarity plasma, increases in luminescence were generally 10 to 25 times greater than that for animals receiving DNA only for all treatment times. This was true for all follow-up days up to day 21. Expression was reduced on day 30. Plasma delivery resulted in statistically significant luminescence levels relative to DNA injection alone for all treatment times up to and including day 14 . On day 21 , the shortest duration treatment, $0.5 \mathrm{~min}$, was no longer significantly increased. On day 30 , only a single treatment time was significant.

Similar-fold increases in luminescence and durations resulted from delivery using negative plasma. Luminescence data from all treatment times were statistically greater than those for DNA injection only on follow-up days $2,4,7,14$, and 21 . The only exception was the 0.5 -min treatment time, which did not result in significantly greater expression. On day 30 , only the two highest treatment times resulted in significantly higher luminescence.

The goal of delivering luciferase was to arrive at conditions that resulted in high expression and then to subsequently use those parameters to deliver EPO encoding DNA. For both positive and negative plasma, no additional benefit occurred when using more than 2 min of plasma exposure to augment delivery. Therefore, a 2-min plasma treatment time was selected. Animals were observed during all delivery procedures, and 


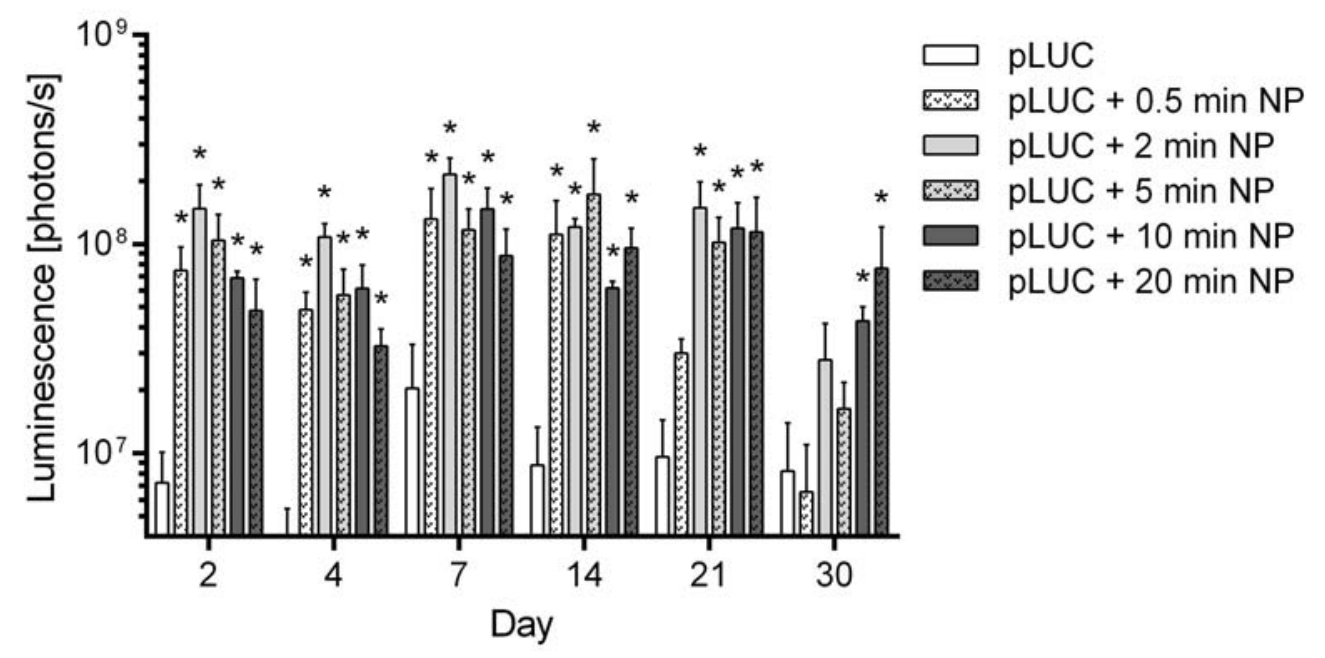

FIG. 4: Long-term luminescence data for animals treated with negative polarity plasma used for DNA delivery. pLUC, Plasmid encoding luciferase administered intradermally; NP, negative plasma; $x x$ min, treatment time with plasma; $n=16$ animals per treatment group. Error bars represent the standard error of the mean. *Statistical significance relative to pLUC injection alone on the same follow-up day

involuntary muscle contractions were not observed during plasma treatment. In addition, treatment sites were assessed visually for several days after plasma exposure; no adverse effects were observed. These observations are in agreement with those of past studies. ${ }^{17-20}$

\section{B. Delivening EPO Encoding Plasmid and Biological Response}

We tested the capacity of plasma to deliver DNA encoding a secreted protein to skin using five groups of animals. Groups were divided into no treatment; DNA injection alone; and DNA injection followed by electroporation, positive polarity plasma, or negative polarity plasma. A 2-min plasma treatment time was used for both positive and negative polarity plasma. Treatment was performed on day 0 . Ten days before treatment, blood was drawn to determine baseline levels of EPO and hemoglobin. Figure 5 shows EPO levels of all treatment groups on days 10, 20, 30, and 40 post-treatments. EPO levels in the no-treatment group were close to the lower limit of detection. However, a measurable increase resulted in the groups that received injection of DNA alone as well as injection followed by electroporation or plasma treatment. For all follow-up days, EPO levels resulting from electroporation and positive polarity plasma were statistically higher than those resulting from DNA injection alone. Hemoglobin results, shown in Fig. 6, were similar. Statistically significant increases, relative to plasmid injection alone, resulted from delivery with electroporation and positive plasma. These results were significant for all four follow-up time points. 


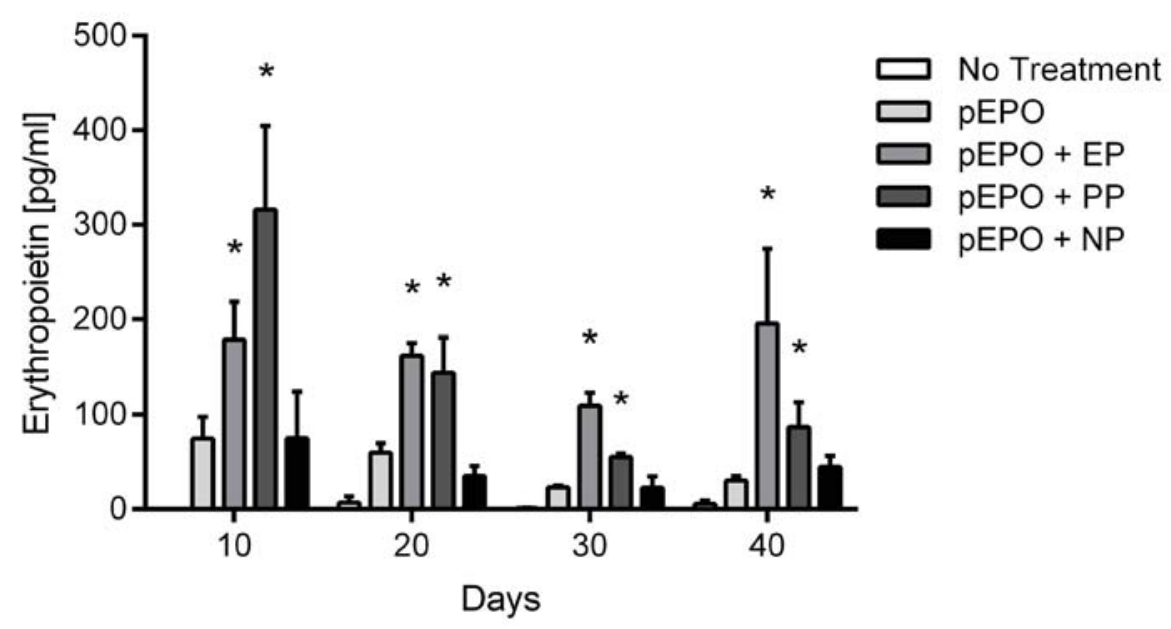

FIG. 5: EPO levels in animals that had delivery of plasmid encoding murine EPO. No treatment, no DNA; pEPO, injection of murine EPO encoding plasmid; EP, electroporation with MEA; PP, positive polarity plasma treatment for $2 \mathrm{~min}$; NP, negative polarity treatment for $2 \mathrm{~min} ; n=12$ animals per group. Error bars represent the standard error of the mean. *Statistical significance relative to $\mathrm{pEPO}$ injection alone on the same follow-up day.

\section{IV.DISC USSION}

When plasmid DNA injection into the dermis is coupled with a physical delivery method, expression can be greatly enhanced. Methods such as electroporation have been reliable and effective tools for delivery; however, their contact-dependent nature often results in undesirable effects. Plasma-based delivery offers enhanced expression without skin contact. Plasma uses a stream of charge deposited on the surface of the skin, which should result in electric fields within the tissue. Thus, the mechanism of action for inducing plasmid DNA uptake by cells is thought to be similar to electroporation.

This appeared to be the case, because increased EPO was detected in circulating blood distant from the treatment site, signifying that local/epidermal EPO secretion occurred in an endocrine-like fashion. The resulting changes in hemoglobin levels indicate that secreted EPO was functional. These results support the use of localized plasmamediated gene delivery to generate an effective systemic response. Conceptually, this study shows that the delivery method should result in the natural mode of action for a secreted protein when the gene for that protein has been delivered to, translated by, and secreted by an atypical yet autologous cell population.

\section{ACKNOWEDGMENT}

This project was supported, in part, by Grant No. AI090561 from the National Institute of Allergy and Infectious Diseases (NIAID). Its contents are solely the responsibility of the authors and do not necessarily represent the official views of the NIAID.

Volume 7, Issue 3, 2017 


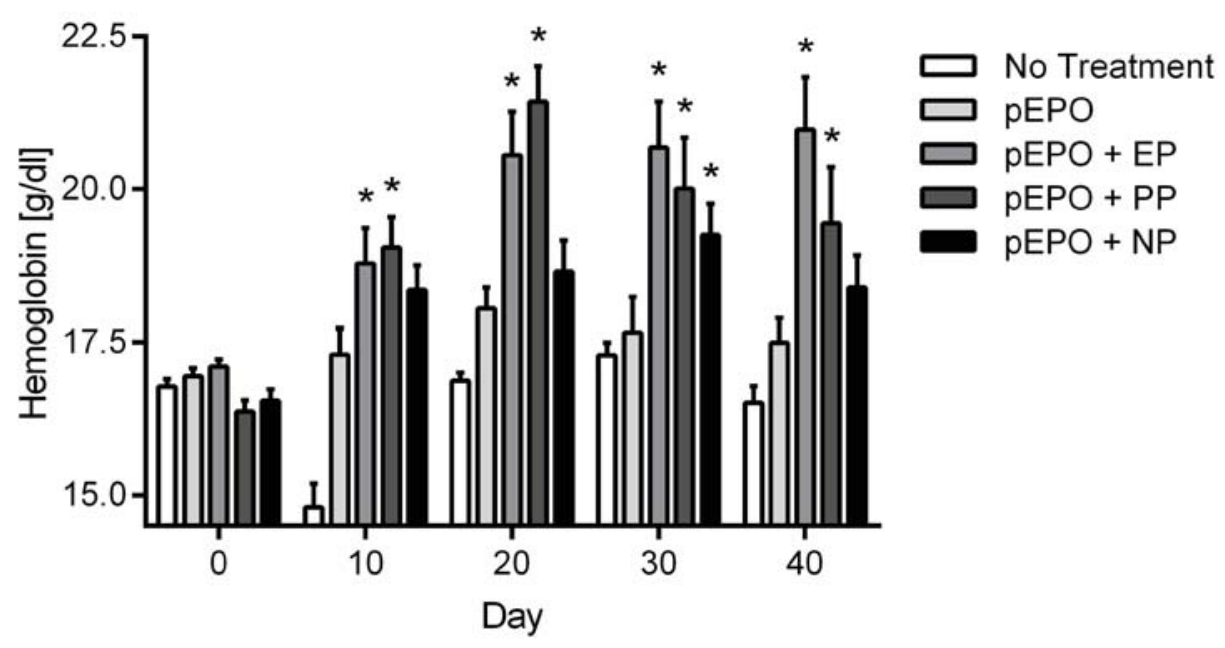

FIG. 6: Hemoglobin levels in animals that had delivery of plasmid encoding murine EPO. Data are from same animals as those in Fig. 5. No treatment, no DNA; pEPO, injection of murine EPO encoding plasmid; EP, electroporation with MEA; PP, positive polarity plasma treatment for 2 min; NP, negative polarity treatment for $2 \mathrm{~min} ; n=12$ animals per group. Error bars represent the standard error of the mean. *Statistical significance relative to $\mathrm{pEPO}$ injection alone on the same follow-up day.

\section{REFERENCES}

1. Lambricht L, Lopes A, Kos S, Sersa G, Preat V, Vandermeulen G. Clinical potential of electroporation for gene therapy and DNA vaccine delivery. Expert Opin Drug Deliv. 2016;13(2):295-310.

2. Heller R, Heller LC. Gene electrotransfer clinical trials. Adv Genet. 2015;89:235-62.

3. Mir LM. Nucleic acids electrotransfer-based gene therapy (electrogenetherapy): Past, current, and future. Mol Biotechnol. 2009;43(2):167-76.

4. Rols MP, Teissie J. Electropermeabilization of mammalian cells. Quantitative analysis of the phenomenon. Biophys J. 1990;58(5):1089-98.

5. Neumann E, Toensing K, Kakorin S, Budde P, Frey J. Mechanism of electroporative dye uptake by mouse B cells. Biophys J. 1998;74(1):98-108.

6. Weaver JC, Chizmadzhev YA. Theory of electroporation: A review. Bioelectrochem Bioenerg. 1996;41(2):135-60.

7. Rols MP. Mechanism by which electroporation mediates DNA migration and entry into cells and targeted tissues. In: Li S, editor. Electroporation protocols: Preclinical and clinical gene medicine. Totowa, NJ: Humana Press; 2008. p. 19-33.

8. Golzio M, Escoffre JM, Portet T, Mauroy C, Teissie J, Dean DS, Rols MP. Observations of the mechanisms of electromediated DNA uptake_-From vesicles to tissues. Curr Gene Ther. 2010;10(4):256-66.

9. Prausnitz MR. The effects of electric current applied to skin: A review for transdermal drug delivery. Adv Drug Deliv Rev. 1996;18(3):395-425.

10. Tjelle TE, Salte R, Mathiesen I, Kjeken R. A novel electroporation device for gene delivery in large animals and humans. Vaccine. 2006;24(21):4667-70.

11. Daud AI, DeConti RC, Andrews S, Urbas P, Riker AI, Sondak VK, Munster PN, Sullivan DM, Ugen KE, Messina JL, Heller R. Phase I trial of interleukin-12 plasmid electroporation in patients with metastatic melanoma. J Clin Oncol. 2008;26(36):5896-903. 
12. Edelblute CM, Heller LC, Malik MA, Heller R. Activated air produced by shielded sliding discharge plasma mediates plasmid DNA delivery to mammalian cells. Biotechnol Bioeng. 2015;112(12): 2583-90.

13. Connolly RJ, Lopez GA, Hoff AM, Jaroszeski MJ. Characterization of plasma mediated molecular delivery to cells in vitro. Int J Pharm. 2010;389(1-2):53-7.

14. Ogawa Y, Morikawa N, Ohkubo-Suzuki A, Miyoshi S, Arakawa H, Kita Y, Nishimura S. An epoch-making application of discharge plasma phenomenon to gene-transfer. Biotechnol Bioeng. 2005;92(7):865-70.

15. Ramachandran N, Jaroszeski M, Hoff AM. Molecular delivery to cells facilitated by corona ion deposition. IEEE Trans Nanobiosci. 2008;7(3):233-9.

16. Stoffels E, Kieft IE, Sladek REJ, van den Bedem LJM, van der Laan EP, Steinbuch M. Plasma needle for in vivo medical treatment: Recent developments and perspectives. Plasma Sources Sci Technol. 2006;15(4):S169.

17. Connolly RJ, Rey JI, Lambert VM, Wegerif G, Jaroszeski MJ, Ugen KE. Enhancement of antigen specific humoral immune responses after delivery of a DNA plasmid based vaccine through a contactindependent helium plasma. Vaccine. 2011;29(39):6781-4.

18. Connolly RJ, Chapman T, Hoff AM, Kutzler MA, Jaroszeski MJ, Ugen KE. Non-contact helium-based plasma for delivery of DNA vaccines. Hum Vacc Immunother. 2012;8(11):1729-33.

19. Connolly RJ, Hoff AM, Gilbert R, Jaroszeski MJ. Optimization of a plasma facilitated DNA delivery method. Bioelectrochemistry. 2015;103:15-21.

20. Connolly RJ, Lopez GA, Hoff AM, Jaroszeski MJ. Plasma facilitated delivery of DNA to skin. Biotechnol Bioeng. 2009;104(5):1034-40.

21. Edelblute CM, Heller LC, Malik MA, Bulysheva A, Heller R. Plasma-activated air mediates plasmid DNA delivery in vivo. Mol Ther Meth Clin Dev. 2016;3:16028.

22. Shah K, Connolly RJ, Chapman T, Jaroszeski MJ, Ugen KE. Electrogenetherapy of B16.F10 murine melanoma tumors with an interleukin-28 expressing DNA plasmid. Hum Vaccin Immunother. 2012;8(11):1722-8.

23. Heller R, Cruz Y, Heller LC, Gilbert RA, Jaroszeski MJ. Electrically mediated delivery of plasmid DNA to the skin, using a multielectrode array. Hum Gene Ther. 2009;21(3):357-62.

24. Guo S, Donate A, Basu G, Lundberg C, Heller L, Heller R. Electro-gene transfer to skin using a noninvasive multielectrode array. J Control Rel. 2011;151(3):256-62.

25. Gothelf A, Hojman P, Gehl J. Therapeutic levels of erythropoietin (EPO) achieved after gene electrotransfer to skin in mice. Gene Ther. 2010;17(9):1077-84. 